\title{
Electronic commerce recommendation mobile crowd system based on cooperative data collection and embedded control
}

\author{
Juan Wang ${ }^{*}$, Wen-Min Deng and Xing-Yue Yin
}

\begin{abstract}
It is known that the collection of the specific needs of mobile users and location management in an electronic commerce recommendation system are important indicators used to evaluate user satisfaction and system execution efficiency. In order to improve the low accuracy of recommendation systems and ensure real-time location management, we proposed cooperative embedded data collection and control of the electronic commerce recommendation system for mobile crowd system. First of all, we would assign every user with a mobile crowd terminal. The terminal could collect the user's demand and personalized data. An embedded terminal could provide users with a real-time, accurate, personalized shopping experience. The terminal could collect the user demand dynamic evolution function. Then, the electronic commerce recommendation application platform would be formed through the deployment of a mobile crowd terminal. The platform collects and forwards the signal acquisition through a single port, dual port and port more adaptive network structure. Experimental results show that the performance of the proposed scheme is superior to the electronic commerce recommendation system based on user personalization drive, such as the data collection of the embedded crowd terminal delay, precision and accuracy of electronic commerce recommendation, as well as the number of iterations. The proposed scheme is more suitable for an electronic commerce recommendation system.
\end{abstract}

Keywords: Cooperative data collection, Electronic commerce recommendation, Embedded control, Mobile crowd

\section{Introduction}

With the development of mobile Internet and electronic commerce (e-commerce), trading volumes soared. The structure of the e-commerce system is complex [1] and providing users with a large amount of commodity information can lead to a bottleneck. E-commerce recommendation system [2] can communication with users and percept the user demand for providing powerful purchase guarantee. However, e-commerce recommendation system has the poor ability of the user demand perception, data collection and system management[3].

The mobile crowd system has been used by researchers to provide data support for e-commerce recommendation. Zhao et al. [4] presented the three-layer framework for mobile data collection in wireless sensor networks, which includes the sensor layer, cluster head layer, and mobile

\footnotetext{
* Correspondence: wjuancn@sina.com

Management School of Nanjing University of Posts and Telecommunications, Nanjing 210023, China
}

collector layer. Ye et al. [5] proposed a context-aware model combined with a crowd-sensing paradigm to achieve fine-grained measurement of a user's current context.

Here, our key issue is the improvement of the data collection of user e-commerce requirements. Velmani and Kaarthick [6] proposed the Velocity Energy-efficient and Link-aware Cluster-Tree (VELCT) scheme for data collection in wireless sensor networks (WSNs), which would effectively mitigate the problems of coverage distance, mobility, delay, traffic, tree intensity, and endto-end connection. An Asynchronous Distributed Data Collection algorithm with fairness consideration for cognitive radio networks (CRNs) was proposed by Zhipeng et al. [7]. Simplicio et al. [8] proposed the SecourHealth, which is a lightweight security framework focused on highly sensitive data collection applications. Zhaosheng et al. [9] proposed the multi-threshold control repair method to clean and repair the probe vehicle
照 Springer

(c) 2016 Wang et al. Open Access This article is distributed under the terms of the Creative Commons Attribution 4.0 International License (http://creativecommons.org/licenses/by/4.0/), which permits unrestricted use, distribution, and reproduction in any medium, provided you give appropriate credit to the original author(s) and the source, provide a link to the Creative Commons license, and indicate if changes were made. 
data. Lu et al. [10] used game theoretic analysis based on coding-aware peer-to-peer data repair in multi-rate wireless networks.

The rest of the paper is organized as follows. Section 2 describes the cooperative data collection mechanism in the e-commerce system. In Section 3, we describe the mobile crowd system for e-commerce recommendation with embedded control. Simulation results are given in Section 4. Finally, we conclude the paper in Section 5.

\section{Cooperative data collection mechanism in the e-commerce system}

To provide a user with a real-time accurate e-commerce recommendation system and personalized shopping experience, its dynamic evolutionary process requires collection of the user's specific needs and mobility management and configuration for each user with a user's demand and personalized data collection of mobile terminal and its application.

The architecture of the e-commerce recommendation intelligence network between the server and the user deployment of the mobile group is shown in Fig. 1. Each user is assigned a custom mobile group of terminal equipment. The following three aspects verification would be made before connecting e-commerce recommendation system with the users or mobile crowd terminal. After verification is completed, the user is connected to the data exchange center. Data exchange center control center through the e-commerce to connect to the server farm. Data exchange center control would connect with server group through the ecommerce control center. These data are made by the user's mobile crowd terminal acquisition.Recommend computing center would make data mining and directly return the results to the data exchange center.

The e-commerce recommendation system user scale has dynamic uncertainties that are hard to predict; therefore, the need to deploy mobile crowd terminal equipment has to be adjusted in real time. Mobile terminal equipment scale can be calculated by Equation (1).

$$
N_{M C}=N \frac{D_{S C}}{d_{c}} C_{e}^{S_{G}}
$$

In Equation (1), $\mathrm{N}_{\mathrm{MC}}$ represents mobile group of terminal size, $\mathrm{N}$ represents user scale, $\mathrm{D}_{\mathrm{SC}}$ represents

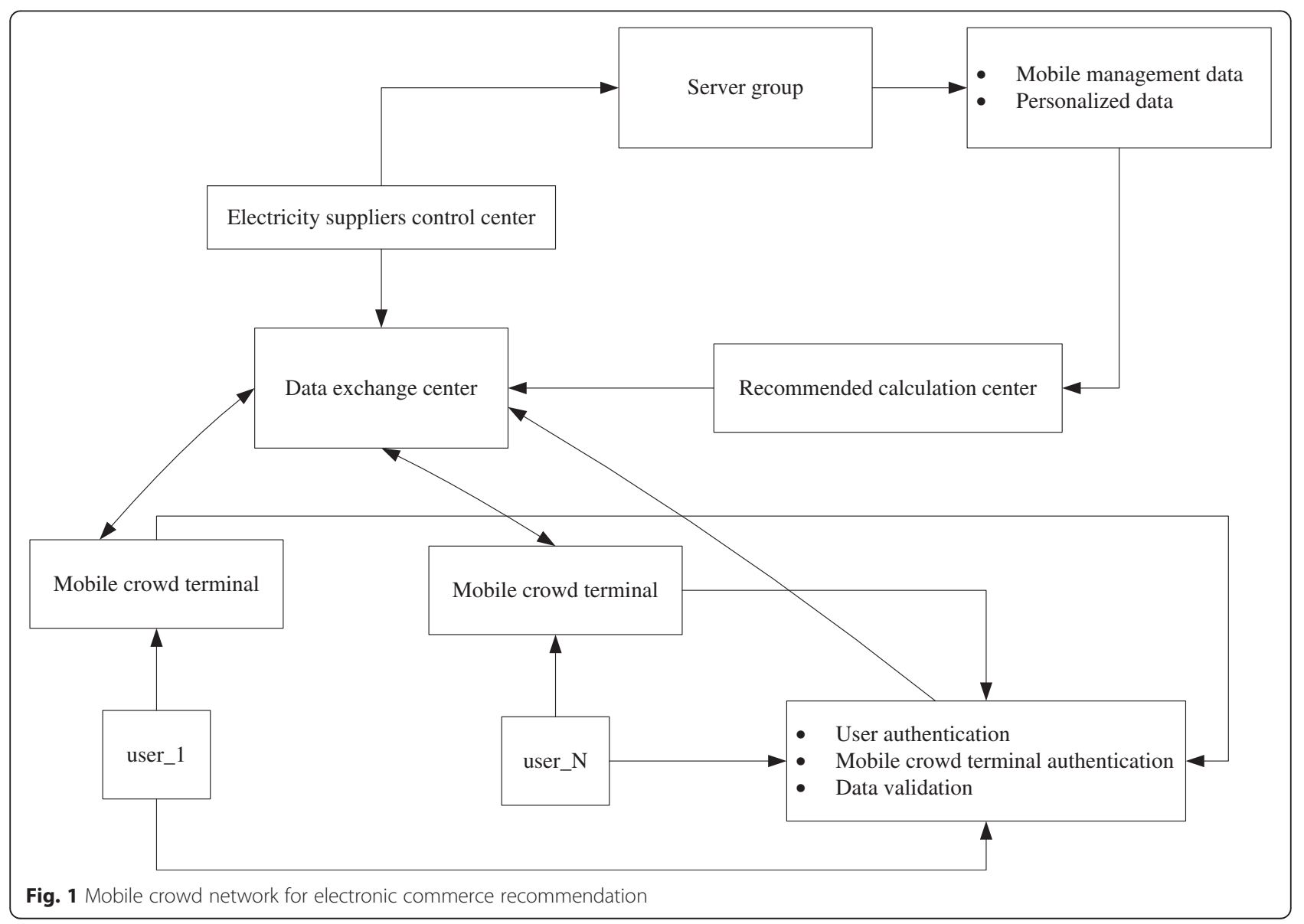


user-generated data size, $d_{c}$ represents mobile group of terminals and data exchange center distance, $\mathrm{C}_{\mathrm{e}}$ represents the server group of computing power and recommend computing center, and the space gain of $\mathrm{S}_{\mathrm{G}}$ represents computing center.

Mobile crowd terminal should be carried out according to the laws of the user data to produce adaptive state adjustment, according to Equation (2) to realize the adaptive optimization. The mobile crowd terminal state is defined as active, dormant, leisure, and metastasis, which are expressed as $0,1,2$ and 4 , respectively. The state transition process is shown in Fig. 2.

$$
S T_{M C}=\left\{\begin{array}{l}
0, D_{S C} \geq D_{T H} \mid N_{M C} \leq N \\
1, D_{S C} \geq D_{T H} \mid N_{M C} \geq N \\
2, D_{S C} \leq D_{T H} \mid N_{M C}>>N \\
4, D_{S C}<<D_{T H} \mid N_{M C} \geq N
\end{array}\right.
$$

In Equation (2), the $\mathrm{D}_{\mathrm{TH}}$ is the collecting data threshold of mobile terminal.

There are four transfer drive processes: State detection, Passive transfer, Target state decision, and Active transfer. Here, the neighbor terminals would transfer the state cooperatively when the mobile crowd terminals have collected the data. The following points should be noted in cooperative data collection of mobile crowd terminal state transition:
(1) State detection. If the number of $1 \mathrm{ST}_{\mathrm{MC}}$ in the binary representation is greater than 2 in STMC, there is an error.

(2) Transfer can be divided into passive and active.

(3) To transfer to the target state.

(4) If the target state is unable to be implemented, the best passive transfer target state would be selected through cooperative data collection mechanism.

(5) Initiative to transfer factor including data size and active mobile terminal size.

(6) When mobile crowd terminal is in a state of "transfer", the mobile crowd system can actively enter the active state.

(7) Transfers of active, dormant and idle states have linear one-way characteristics.

(8) Only idle mobile crowd system can enter a state of transfer and join the cooperative data collection.

\section{Mobile crowd system for e-commerce recommendation with embedded control}

Several problems have to be solved to apply mobile crowd system in the process of e-commerce recommendation as follows:

(1) How to design a mobile embedded terminal which can collect the user's personalized needs.

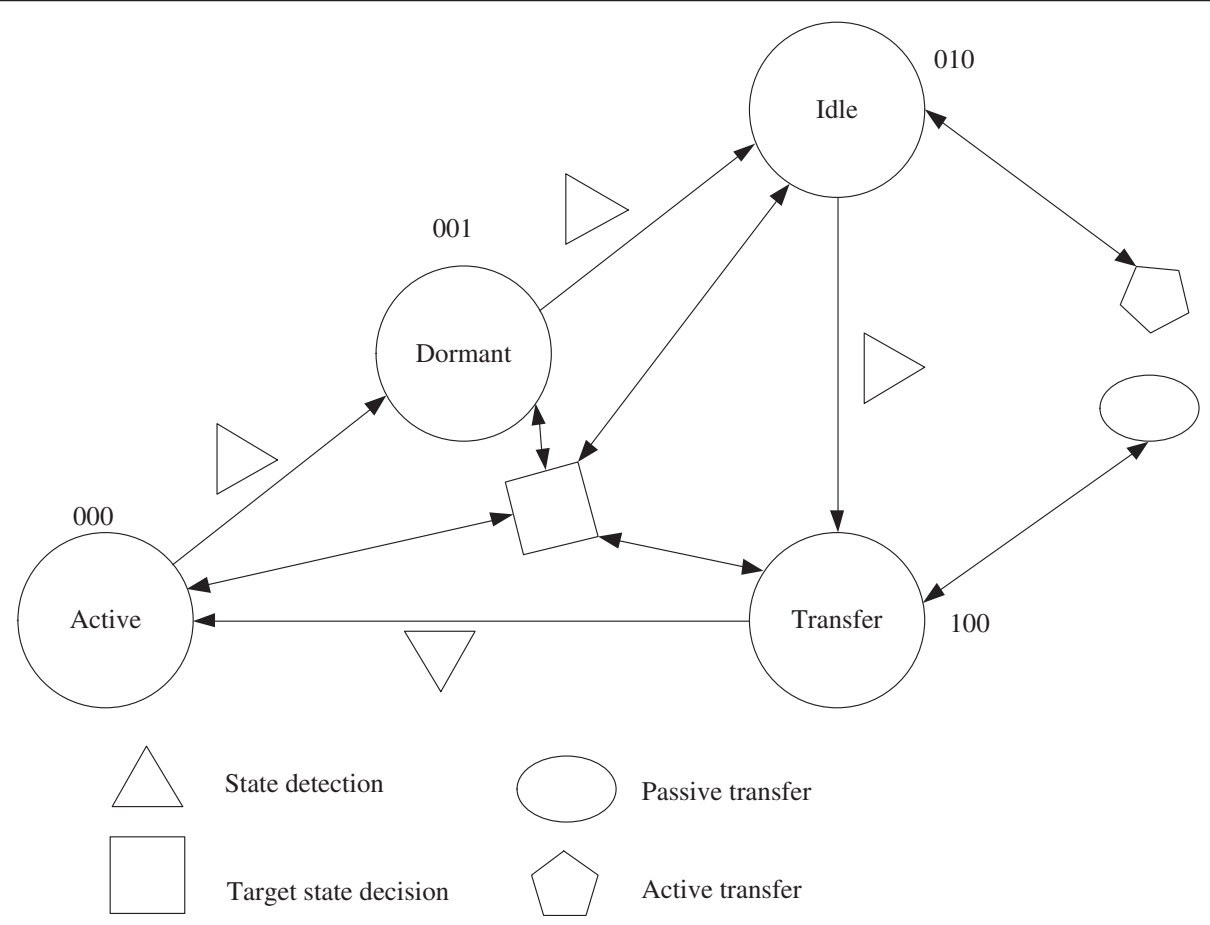

Fig. 2 State transfer of mobile crowd terminal 
(2) How to realize the service launch and accept the e-commerce recommendation.

(3) How to monitor the related factors that affect e-commerce recommendation service. Based on the scheme, the system has the function of intelligent control and restoration.

Figure 3 mobile group of terminals shows recommended application in e-commerce deployment. The terminal has a single port, dual port and port of signal acquisition and forwarding network structure, as shown in Fig. 4. According to the relationship shown in Equation (3), the mobile group of intelligent equipment can choose adaptive port number. Different ports collect a user's personalized needs. On this basis, the user's future demands can be predicted by Equation (4).

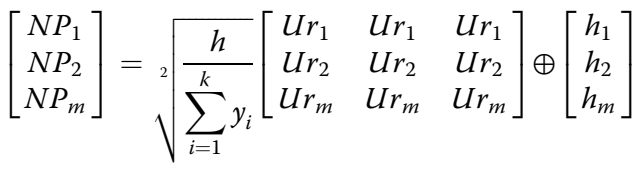

In Equation (3), NP represents embedded mobile group of terminal network port, $\mathrm{H}$ represents the mean wireless channel quality, y represents multipath signal power and transmission, and $U r$ is user requirements.

$$
\begin{aligned}
{\left[\begin{array}{c}
U r_{1} \\
\vdots \\
U r_{n}
\end{array}\right]=} & {\left[\begin{array}{ccc}
U r^{1}{ }_{1} & \cdots & U r^{k}{ }_{1} \\
\vdots & \vdots & \vdots \\
U r^{1}{ }_{n} & \cdots & U r^{k}{ }_{n}
\end{array}\right] } \\
& *\left\{\begin{array}{c}
\alpha_{1} \sum_{i=1}^{k} U r^{i}{ }^{i} \\
\vdots \\
\alpha_{n} \sum_{i=1}^{k} U r_{1}^{i}
\end{array}\right.
\end{aligned}
$$

In Equation (4), $\mathrm{k}$ represents mobile group of intellectual equipment port number, alpha predictor represents user requirements, and $\mathrm{N}$ represents user scale.

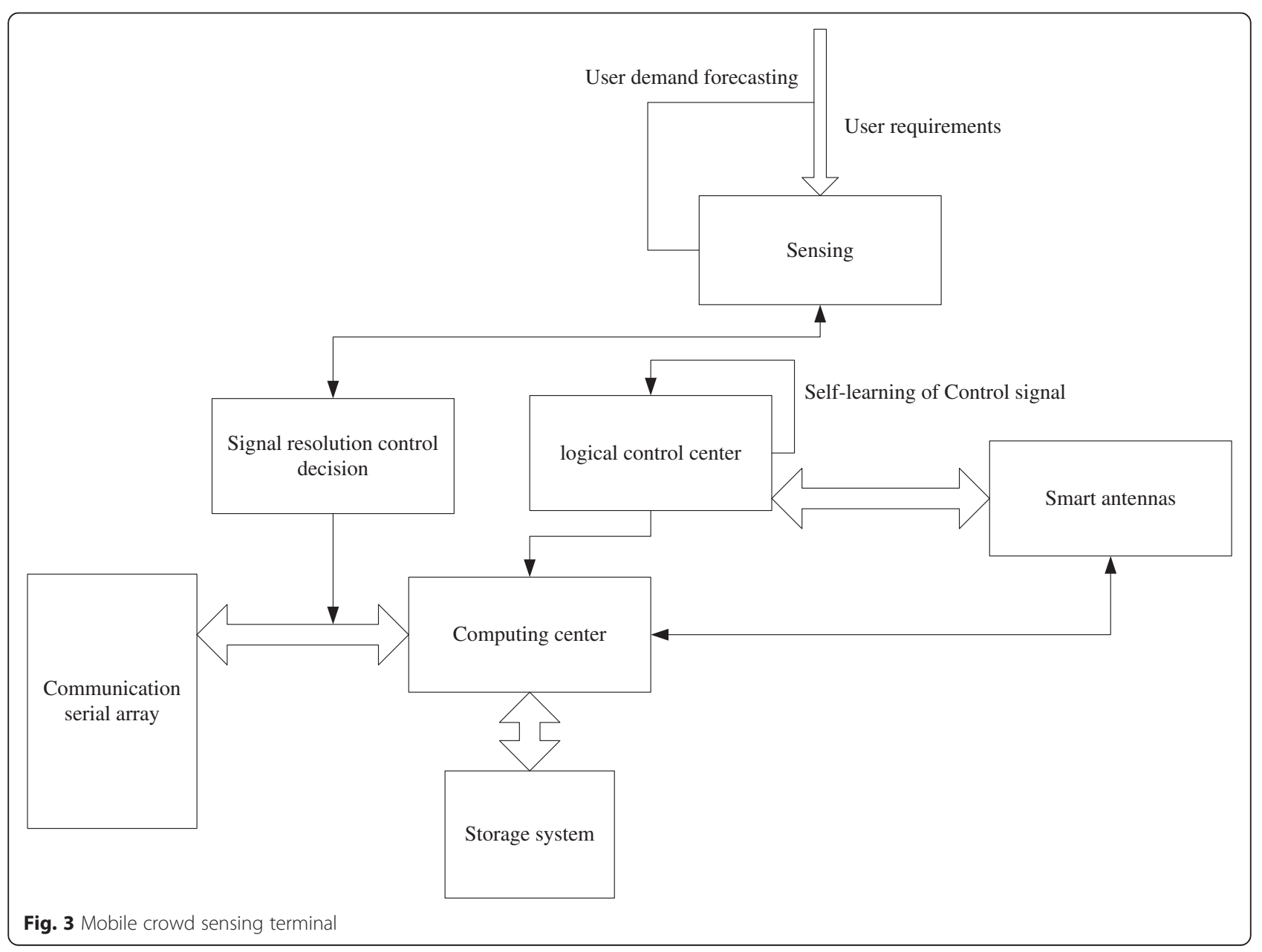




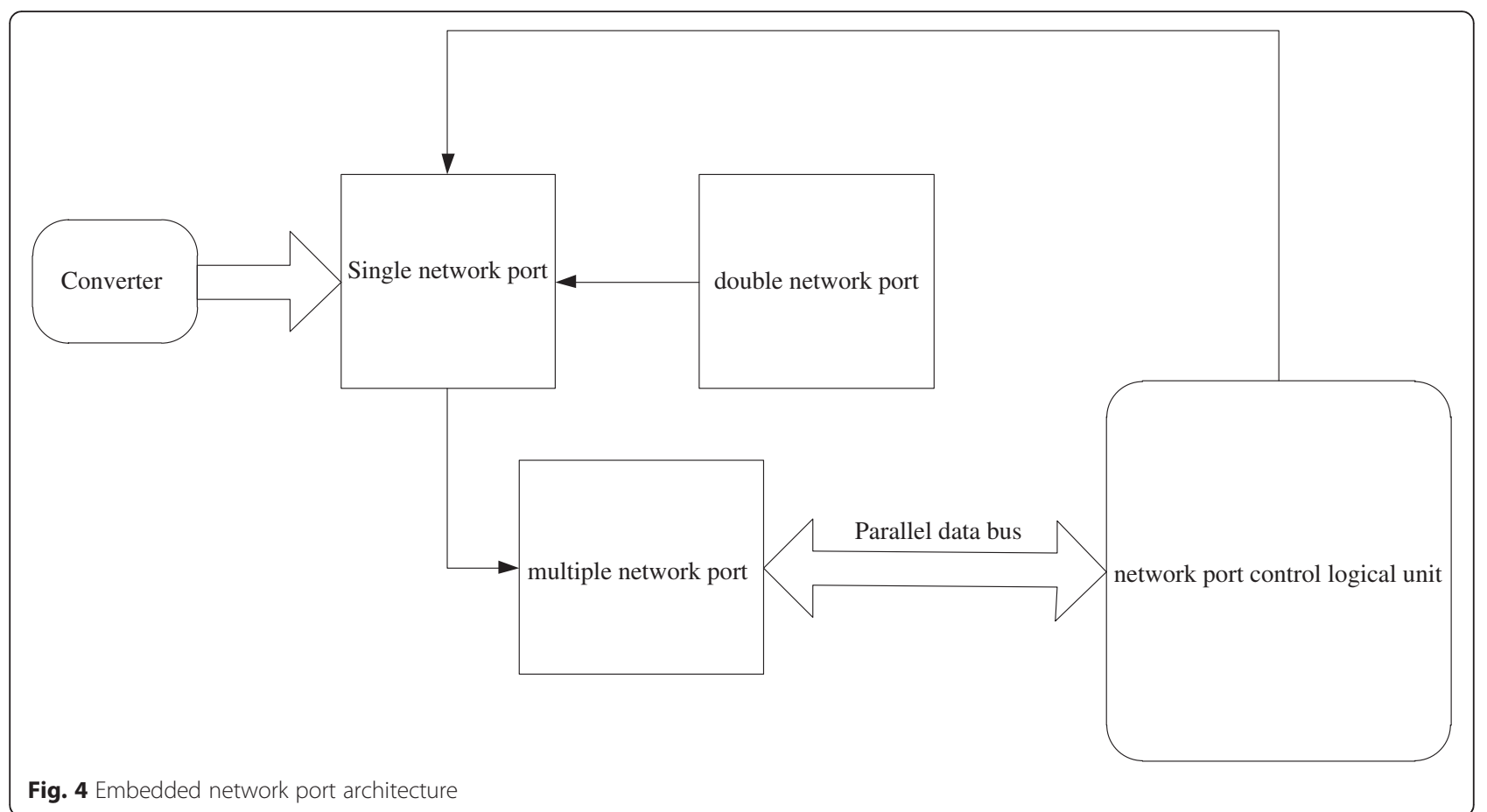

\section{Performance analysis and verification}

In this section, we verify the performance of the proposed solution (ECR - CD), from the embedded intelligent terminal data collection delay, precision and accuracy of e-commerce recommendation and iteration number; at the same time, based on user driven personalized e-commercerecommendation system (CR - UR) were compared. Performance indicators are defined as follows:

(1) The embedded intelligent terminal data collection delay.

The way time needed for data collection through collaboration between the embedded intelligent terminals, including collaboration delay overhead and data fusion computational overhead.

(2) Data collection of the embedded intelligent terminal data precision.

Forward embedded intelligent terminal to the e-commerce system of data and user experience data contrast.

(3) The e-commerce recommendation accuracy. E-commerce recommendation system would make the users ordering scheme for satisfying the actual user requirements.

(4) The e-commerce recommended number of iterations.

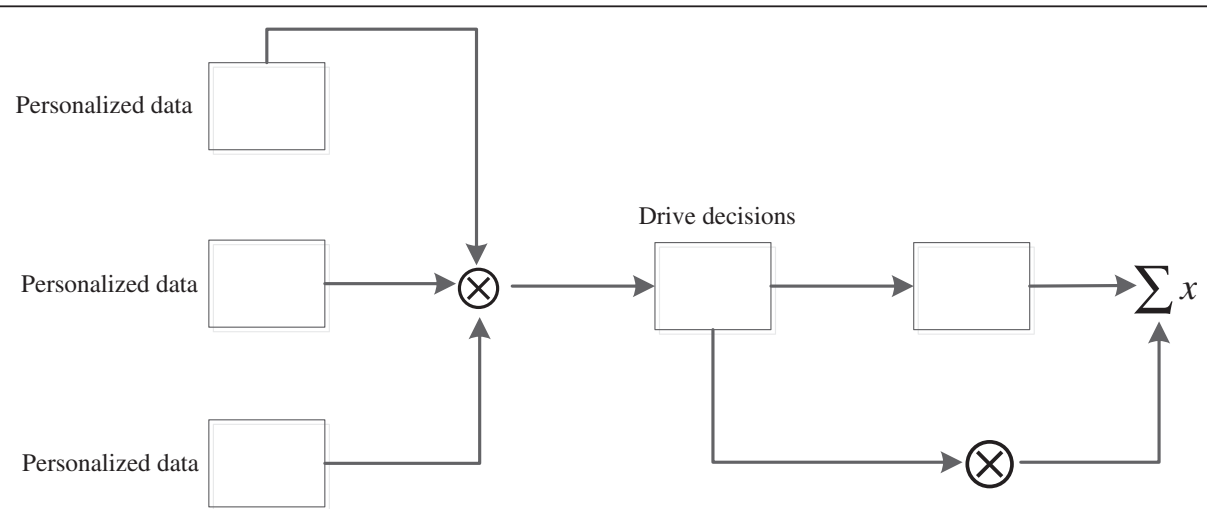

Fig. 5 The encoding process of e-commerce recommendation in thee user driven personalized e-commercerecommendation system 


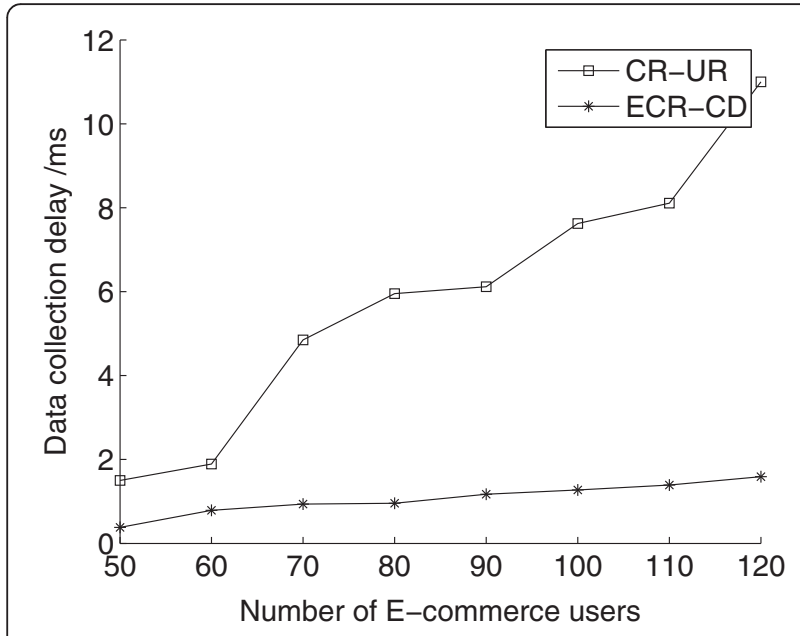

Fig. 6 Embedded intelligent terminal data collection delay

It is the recommend iteration number of completing e-commerce recommendation user requirements.

Based on user personalization drive, Fig. 5 gives the e-commerce recommendation terminal coding scheme of e-commerce recommendation system.

The participants of our experiment were undergraduate and graduate students of Nanjing University of Posts and Telecommunications. We obtained our experimental data of the personalized e-commerce recommendation conclusion through a network investigation and questionnaire survey. At the same time, personalized user demand data from 10 professionals from the liberal arts, sciences and engineering were gathered for comparison.

Through the processes shown in Figs. 3 and 4, we obtain the ECR - CD package data. The verification and comparison results are shown in Fig. 6, 7, 8 and 9. From Fig. 6, the

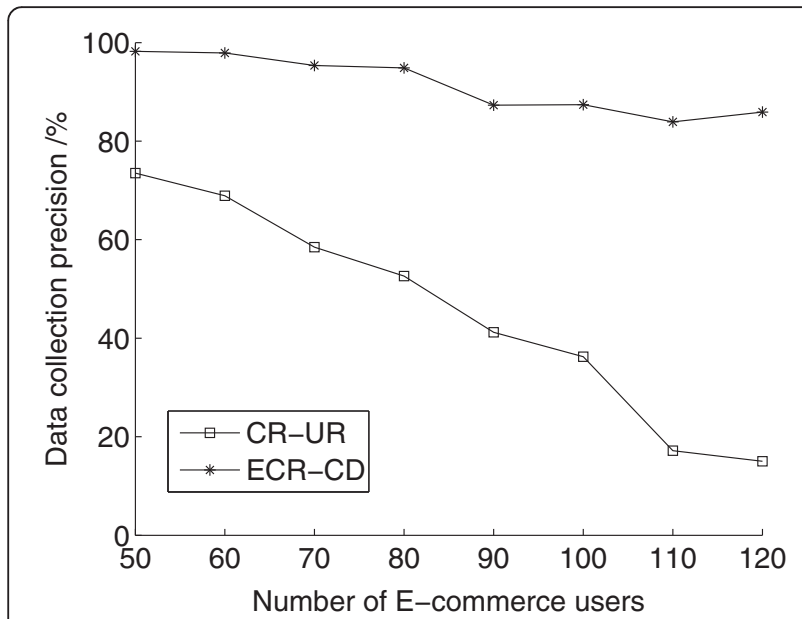

Fig. 7 Data collection precision of the embedded intelligent terminal

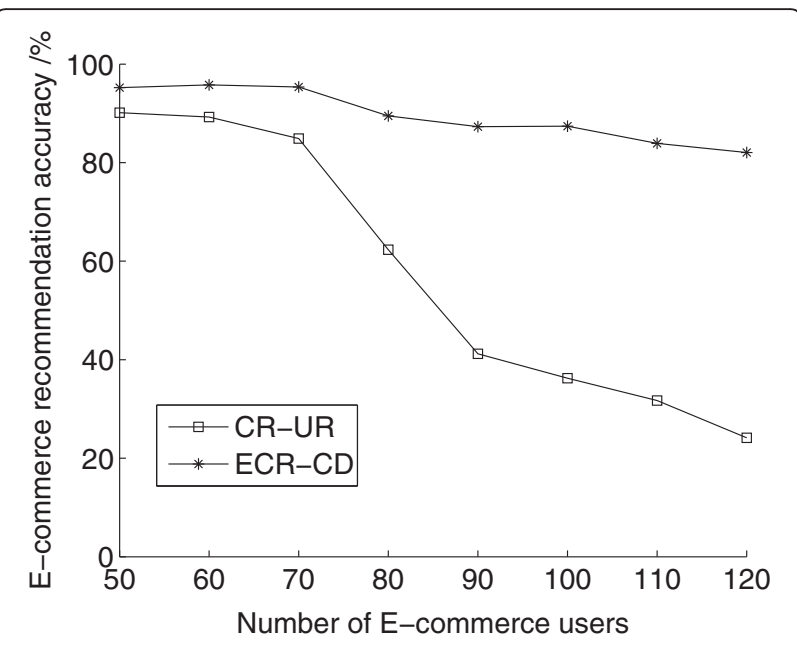

Fig. 8 E-commerce recommendation accuracy

data collection delay of the proposed ECR - CD scheme is smaller than one of the CR - UR scheme. The delay of the proposed ECR - CD scheme is not only smooth but also low. This is because the cooperation process to collect, gather and forward data in the ECR - CD scheme in ecommerce recommendation system deployment of intelligent embedded terminal management scope, led to a short delay. Because the data collection delay was short, most of the time we used a smart terminal for calculations, so data accuracy was higher, as shown in Fig. 7.

Because the mobile embedded terminal of the ECR $\mathrm{CD}$ scheme could obtain the user personalized ecommerce recommendation requirements. At the same time the ECR - CD scheme realized the real time launch and high precision reception of the e-commerce recommendation service. In addition, the scheme can intelligently monitor the related factors that affect the ecommerce recommendation service, and so has realtime intelligent control and the e-commerce

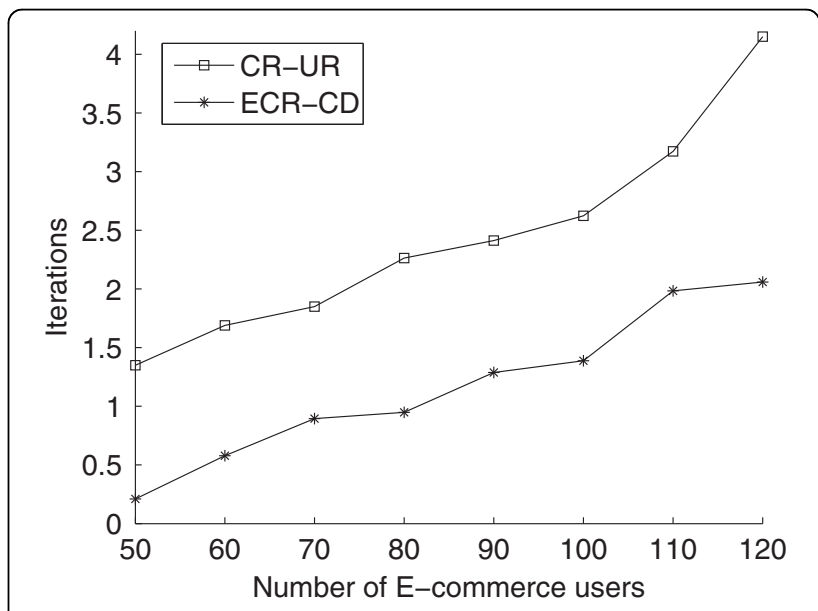

Fig. 9 Electronic commerce recommended number of iterations 
recommendation data repair function has high reliability. Thus the high performance are shown in Fig. 8 and Fig. 9. The ECR - CD scheme not only keep the high recommendation precision of e-commerce but also reduce the iterations number.

\section{Conclusions}

In order to improve the recommend accuracy and real time location management of the e-commerce recommendation system, the mobile crowd e-commerce recommendation system was proposed based on the cooperative embedded data collection and control. The mobile crowd terminal of each user in this system could collect the user demand and the personalized data, which is used to provide accurate real-time user personalized shopping experience and dynamic evolving requirements. There are the collecting and forwarding signal network structure with a single port, dual port and multiple port in the terminal. The experimental results show that the performance of the proposed ECR - CD scheme has obvious advantage, such as the embedded intelligent terminal delay, precision and accuracy of e-commerce recommendation and the iterations number.

\section{Competing interests}

The authors declare that they have no competing interests.

\section{Acknowledgements}

This work is supported in part by the national college students' innovative training program (SZDG2015031), University philosophy social science research project in Jiangsu province education department (2015SJB014), and the fund incubator project of Nanjing University of posts and telecommunications countries (NY214117).

Received: 15 December 2015 Accepted: 29 January 2016 12. (2)

\section{References}

1. S Lin, K Wang, An laboratory experiment for comparing effectiveness of three types of online recommendations. Tsinghua Sci. Technol. 13(3), 293-299 (2008)

2. J Mackiewicz, D Yeats, Product review users' perceptions of review quality: the role of credibility, informativeness, and readability. IEEE Trans. Prof. Commun. 57(4), 309-324 (2014)

3. N Giseop, O Hayoung, L Kyu-haeng et al., Toward trustworthy social network services: A robust design of recommender systems. J. Commun. Netw. 17(2), 145-156 (2015)

4. Miao Zhao; Yuanyuan Yang; Cong Wang. Mobile Data Gathering with Load Balanced Clustering and Dual Data Uploading in Wireless Sensor Networks. IEEE Transactions on Mobile Computing, 14(4), 770-785 (2015)

5. Y Dengpan et al., Mobile crowd-sensing context aware based fine-grained access control mode. Multimedia Tools \& Applications. (2015) doi:10.1007/s1 1042-015-2693-3

6. R Velmani, B Kaarthick, An efficient cluster-tree based data collection scheme for large mobile wireless sensor networks. IEEE Sensors $\mathrm{J}$. 15(4), 2377-2390 (2015)

7. C Zhipeng, J Shouling, $\mathrm{H}$ Jing et al., Distributed and asynchronous data collection in cognitive radio networks with fairness consideration. IEEE Trans. Parallel Distributed Syst. 25(8), 2020-2029 (2013)

8. MA Simplicio, LH Iwaya, BM Barros et al., SecourHealth: A Delay-tolerant security framework for mobile health data collection. IEEE J. Biomed. Health Inform. 19(2), 761-772 (2014)
9. Z Zhaosheng, Y Diange, Z Tao et al., A study on the method for cleaning and repairing the probe vehicle data. IEEE Trans. Intell. Transport. Syst. 14(1), 419-427 (2013)

10. H-C Lu, W Liao, MC Chen et al., Coding-aware peer-to-peer data repair in multi-rate wireless networks: a game theoretic analysis. IEEE J. Selected Areas Commun. 31(9), 391-398 (2013)

\section{Submit your manuscript to a SpringerOpen ${ }^{\circ}$ journal and benefit from:}

- Convenient online submission

- Rigorous peer review

- Immediate publication on acceptance

- Open access: articles freely available online

- High visibility within the field

Retaining the copyright to your article

Submit your next manuscript at $>$ springeropen.com 